\title{
Study on the Correction of $S-N$ Distribution in the Welding Fatigue Analysis Method Based on the Battelle Equivalent Structural Stress by Rough Set Theory
}

\author{
Yibo Sun ${ }^{1,2}$ - Xinhua Yang1,* \\ ${ }^{1}$ Dalian Jiaotong University, School of Material Science and Engineering, China \\ 2 Dalian Jiaotong University, School of EMU Application and Maintenance Engineering, China
}

\begin{abstract}
In welding techniques, failure is a key problem that is related to the stability and safety of the welded structure. In the commonly used welding fatigue analysis methods, the Master S-N curve method based on Battelle structural stress solves the problem of inconsistencies in stress calculation and S-N curve selection better than the nominal stress method and hot spot stress method. In this paper, rough set theory was employed to study the S-N distribution based on the Battelle equivalent structural stress. Firstly, rough set analyses of the S-N distribution based on the nominal stress, structure stress and Battelle equivalent structure stress were carried out. Then the S-N distributions based on the three stresses were studied. The results indicated that structural stress rearranges the S-N point making it much more concentrated in the region near the mean S-N curve and that equivalent structural stress places the S-N point more uniformly on both sides of the curve. Subsequently, the corrections from the plate thickness and stress ratio in the Master S-N curve method were studied. It was concluded that with two steps of corrections the decision-making degrees of welding factors are weakened and harmonized in the Battelle equivalent structural stress. This made the S-N distribution much more concentrated and uniform, which allows more accurate welding fatigue prediction by the Master S-N curve.
\end{abstract}

Keywords: welding fatigue, Battelle equivalent structural stress, Master S-N curve method, rough set theory

\section{INTRODUCTION}

As a traditional processing technique welding has been widely used in many fields, such as mechanical manufacturing, aerospace, transportation, etc. During the welding process, metal in the welding zone fuses at high temperature with its property and geometry changed and thus fatigue damage often occurs in the weld seam, which always determines the life of the welded structure. Therefore the fatigue analysis and life prediction of a welding joint are directly related to the stability and safety of the whole structure.

Currently, the commonly used welding fatigue analysis and prediction methods mainly include the nominal stress method, hot spot stress method and the Master $S$-N curve method based on the Battelle equivalent structure stress. The nominal stress method was first proposed for the fatigue analysis of welded structures [1]. Several standard specifications have been established based on this method using a large amount of experimental data [2] and [3]. While it is widely used in engineering applications, some limitations have gradually come to light, such as that the nominal stress of a complex structure is difficult to determine and that inconsistent stress calculation caused by a singularity in the weld toe also makes the stress value less accurate. In addition, it provides $S-N$ curves in accordance with the classification of the welding joints, which do not always clearly correspond to one type, which limits its universality in engineering applications. The hot spot stress method that obtains the stress in the welding toe by extrapolation based on nominal stress was first proposed by Niemi [4]. It characterizes the stress level in the weld toe by hot spot stress, which makes it available for complex welded structures. In theory it provides a general $S-N$ curve based on the hot spot stress to characterize the fatigue strength of various joints [5]. Although it solves some of the problems of the nominal stress method, but it is difficult to establish a uniform hot type and extrapolation formula for various welding types, which limits the application in engineering.

In 2001, Dong proposed the Master $S-N$ curve method based on the mesh insensitive structural stress (Battelle equivalent structural stress) [7]. In this method, the Battelle equivalent structural stress, which is not sensitive to the mesh size of finite element analysis, is defined, which makes the stress calculation uniform for various mesh sizes. The stress intensity factor correction, taking into account the influences of joint thickness and load model, etc., is then established based on fracture mechanics and the parameters in the formula are ascertained based on a large number of welding fatigue data. Thus a Master $S-N$ curve is obtained to characterize the fatigue life including various welding joint types [8]. The Master $S-N$ curve method is widely applied in many fields and was adopted by ASME as a standard for welding fatigue analysis [9] and [10]. 
In this paper rough set theory was employed to study the $S-N$ data distribution of welding data based on the Battelle equivalent structural stress. $S-N$ curves are established for Titanium alloy welding joints based on nominal stress, structural stress and Battelle equivalent structure stress in the Master $S-N$ curve method. The $S-N$ distributions based on the three stresses were studied. Corresponding to the computational formulas of the Battelle equivalent structural stress, the corrections of $S-N$ data distribution from joint thickness and load ratio in the Battelle equivalent structural stress are also explored.

\section{BASIC PRINCIPLE OF BATTELLE EQUIVALENT STRUCTURAL STRESS}

In the finite element stress calculation the results are affected by mesh size, which causes inconsistency in calculations for different structures. In order to address this problem, structural stress based on the line force is first defined using the Master $S-N$ curve method.

The normal structural stress at each node from elementary structural mechanics theory is given by:

$$
\begin{gathered}
\sigma_{s}=\sigma_{m}+\sigma_{b}, \\
\sigma_{m}=\frac{F_{y}}{A}=\frac{F_{y}}{l \cdot t}, \\
\sigma_{b}=\frac{M_{x}}{W}=\frac{M_{x}}{\frac{1}{6} \cdot l \cdot t^{2}},
\end{gathered}
$$

where $F_{y}$ is the vertical force in the weld toe, $M_{x}$ is the moment around the weld toe. The line force $f_{y}$ and moment $m_{x}$ are defined as $f_{y}=F_{y} / l, m_{x}=M_{x} / l$ as shown in Fig 1.Structural stress can then be expressed as follows [7]:

$$
\sigma_{s}=\sigma_{m}+\sigma_{b}=\frac{f_{y}}{t}+\frac{6 m_{x}}{t^{2}}
$$

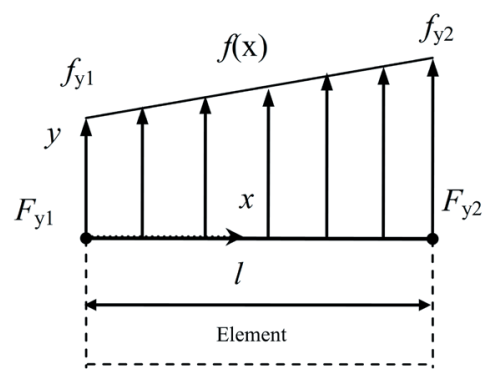

Fig. 1. Definition of line force

While structural stress characterizes the stress state in the weld seam, fracture mechanics is employed to estimate the fatigue life of welding joints. The stress intensity factor in crack propagation theory can be calculated as [11]:

$$
\Delta K=\sqrt{t^{*}}\left[\Delta \sigma_{m} f_{m}(a / t)+\Delta \sigma_{b} f_{b}(a / t)\right],
$$

where $a$ is the crack depth, $t^{*}$ is interpreted as a ratio of actual thickness $t$ to a unit thickness, rendering the term dimensionless. $f_{m}(a / t)$ and $f_{b}(a / t)$ are membrane stress and bending stress as a function of crack growth degree, respectively. Using the Paris crack growth law, the prediction of the lifecycle from an infinitesimally small crack to final failure can be expressed as:

$$
N=\int_{a / t=0}^{a / t=1} \frac{t^{*} d(a / t)}{C\left(M_{k n}\right)^{n}(\Delta K)^{m}}=\frac{1}{C} t^{*\left(1-\frac{m}{2}\right)}\left(\Delta \sigma_{s}\right)^{-m} I(r)
$$

where $M_{k n}$ is the notch stress magnification expressed as $M_{k n}=K / K_{n}$ in which $K$ represents the total $K$ due to both the far-field stress and the local notch stress effects and $K_{n}$ represents only the far-stress contribution to the stress intensity factor. $I(r)$ is a dimensionless function of $r$ and $m$ is the crack growth exponent, which is set to be 3.6 in ASME [9]. A Master $S-N$ curve can be established according to Eq. 6 based on a set of welding fatigue data. Related to Eq. 6 the Battelle equivalent structural stress can be expressed as:

$$
\Delta \sigma_{\varepsilon}=t^{*\left(1-\frac{m}{2}\right)}\left(\Delta \sigma_{s}\right)^{-m} I(r)
$$

where $t^{*}$ is dimensionless the equivalent $\Delta \sigma_{\varepsilon}$ retains a stress unit.

\section{ESTABLISHMENT OF ROUGH SETS ANALYSIS MODEL}

Rough set theory is a mathematical approach that can be employed to handle imprecision, incompletion, vagueness and uncertainty [12]. In this paper the relationship between welding factors and $S-N$ distribution based on nominal stress $(N S)$, structure stress $(S S)$ and Battelle equivalent structure stress $(E S)$ are analyzed using rough set analysis.

Firstly, rough set models were established based on well-documented fatigue data. Titanium alloy low cycle fatigue data for TIG [13], Manual TIG[14], laser welding [15], and various fillet welded joints [16]with different bending versus tension [17] in the as-welded conditions without stress-relief were cited. 106 sets of welding fatigue data were arranged by welding factors including joint type $(J)$, plate thickness $(t)$, load ratio $(R)$, stress ratio $(r)$, welding process $(W)$ and material 
type $(M)$ and entered into the rough set database as condition attributes. The joint types marked with two capital letters were cited from reference [16]. The first letters refer to the directions along $(L)$ and transverse $(C)$ to the plate rolling. The second letters refer to the butt-welded joints $(B)$, transverse fillet welded joints $(T)$ and longitudinal fillet $(L)$ welded joints. Among the parameters $R$ and $r$ were expressed as:

$$
\begin{gathered}
r=\sigma_{b} /\left(\sigma_{m}+\sigma_{b}\right), \\
R=\sigma_{\min } / \sigma_{\max } .
\end{gathered}
$$

The deviation degrees of every $S-N$ data point to the $S-N$ curves based on $N S, S S$ and $E S$ respectively were discretized as decision attributes. The operation is as as shown in Figs. 2 to 4.
According to the nominal stress and the joint geometry in the references, structural stress and Battelle equivalent structural stress were calculated for every set of fatigue data by finite element analysis. With these data the mean $S-N$ curves based on $N S$, $S S$ and $E S$ were fitted using the least square method and the standard deviations (STDEV) were also calculated. The mean $S-N$ curve and deviation curves with -3 to +3 magnifications were then drawn in each coordinate as shown in Figs. 2 to 4.

Seven characteristic curves constituted six regions considered as decision domains which were marked as $D(1)$ to $D(6)$ from left to right. The decision attributes of every set of welding fatigue data were determined by the regions in which the $S-N$ point was found. Figs. 2 to 4 show the $S-N$ curves and data distribution. From the figures it can be seen that fatigue life increases

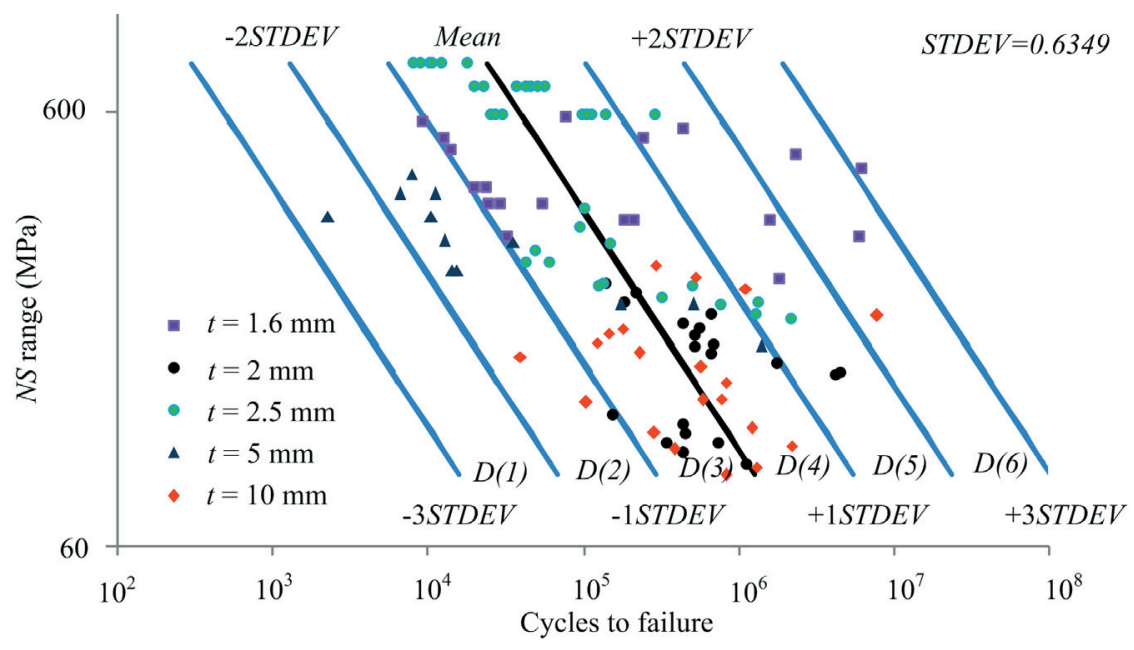

Fig. 2. Decision division of S-N distribution based on NS

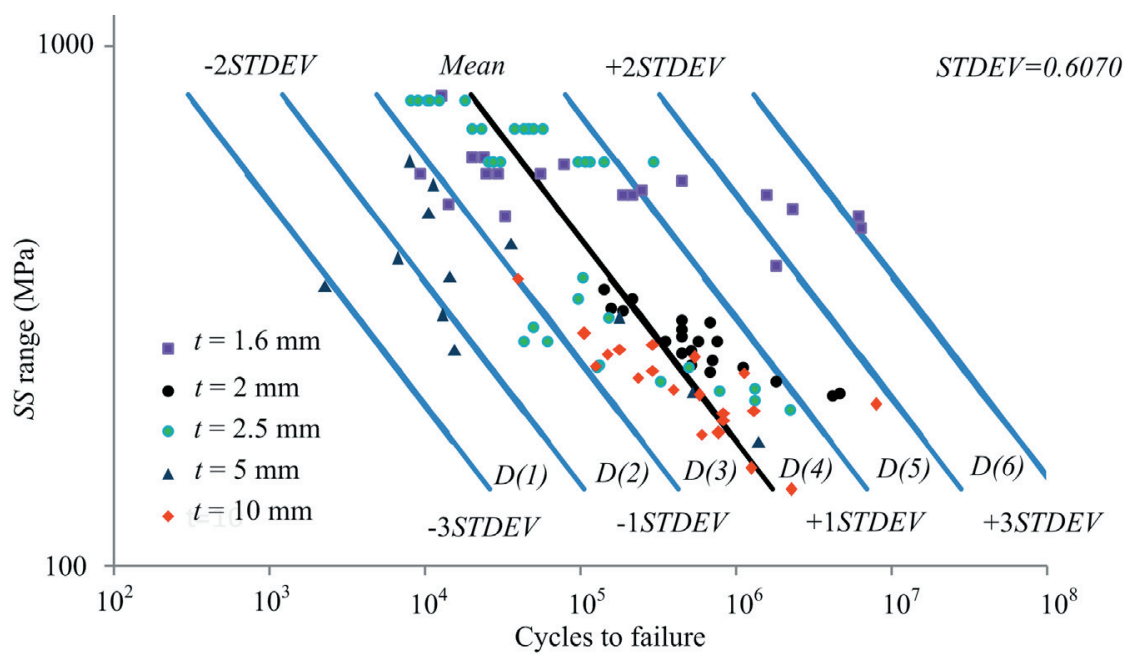

Fig. 3. Decision division of S-N distribution based on SS 


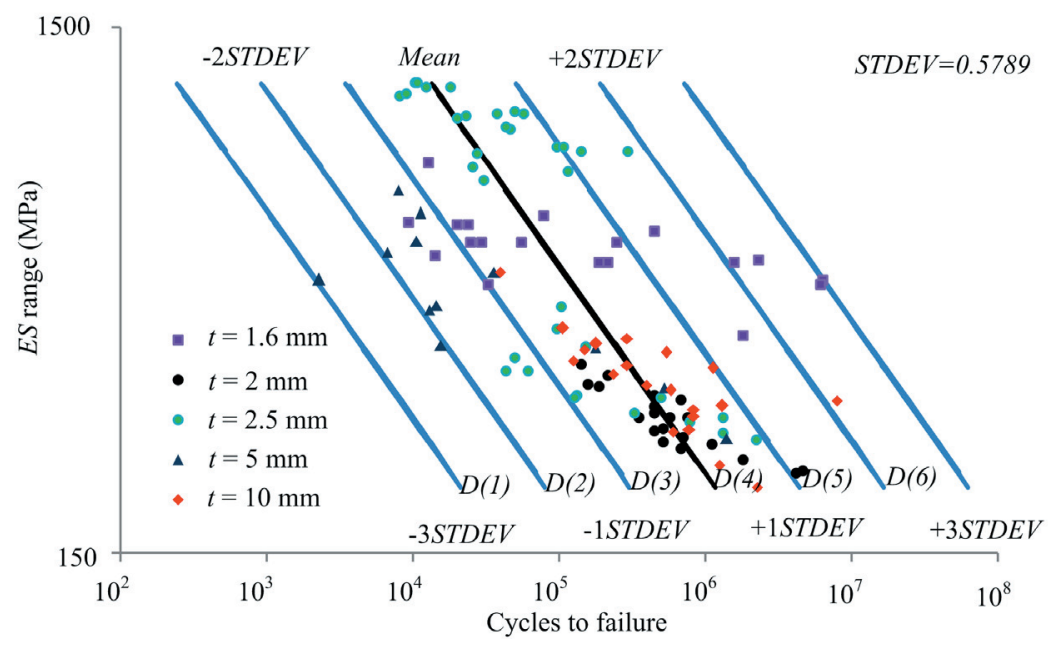

Fig. 4. Decision division of S-N distribution based on ES

Table 1. Parts of the attributes in S-N rough set model based on NS

\begin{tabular}{ccccccc}
\hline & \multicolumn{3}{c}{ Condition attributes } & & Decision attributes \\
\hline$J$ & $t$ & $R$ & $r$ & $W$ & $D$ & 3 \\
\hline LT & 2 & 0 & 0.29 & Manual TIG & JIS H4600 TP340C/H & 3 \\
\hline CT & 2 & 0 & 0.29 & Manual TIG & JIS H4600 TP340C/H & 5 \\
\hline LL & 2 & 0 & 0.34 & Manual TIG & JIS H4600 TP340C/H & 4 \\
\hline CB & 10 & 0 & 0 & Manual TIG & JIS H4600 TP340C/H & 3 \\
\hline CT & 10 & 0 & 0.27 & Manual TIG & JIS H4600 TP340C/H & 6 \\
\hline BUTT & 1.6 & 0.1 & 0 & TIG & Ti-6-2 & TC4 \\
\hline BUTT & 2.5 & 0.1 & 0 & EBW & Ti80 & 3 \\
\hline Cruciform & 5 & 0.1 & 0.27 & Manual TIG &
\end{tabular}

Table 2. Reduction rules of S-N distribution based on NS

\begin{tabular}{cllc}
\hline No. & \multicolumn{1}{c}{ Rules } & Supports \\
\hline 1 & $J(\mathrm{LT})$ and $R(0)$ and $r(0.29)$ and $W(\mathrm{M} \mathrm{Tig})$ and $M(\mathrm{JIS} \mathrm{H} 4600)$ & $\rightarrow D(4)$ or $D(5)$ & $0.83,0.17$ \\
\hline 2 & $J(\mathrm{LL})$ and $R(0)$ and $r(0.34)$ and $W(\mathrm{M}$ Tig) and $M(\mathrm{JISH} 4600)$ & $\rightarrow D(3)$ or $D(2)$ & $0.86,0.14$ \\
\hline 3 & $J(\mathrm{LT})$ and $R(0)$ and $t(2)$ and $W(\mathrm{M}$ Tig) and $M(\mathrm{JISH} H 600)$ & $\rightarrow D(4)$ or $D(5)$ & $0.83,0.17$ \\
\hline 4 & $J(\mathrm{LL})$ and $R(0)$ and $t(2)$ and $W(\mathrm{M}$ Tig) and $M(\mathrm{JISH} H 600)$ & $\rightarrow D(3)$ or $D(2)$ & $0.86,0.14$ \\
\hline
\end{tabular}

Table 3. Reduction rules of S-N distribution based on SS

\begin{tabular}{|c|c|c|c|}
\hline No. & Rules & & Supports \\
\hline 1 & $J(\mathrm{LT})$ and $R(0)$ and $r(0.29)$ and $W(\mathrm{M} \mathrm{Tig})$ and $M(\mathrm{JIS} \mathrm{H} 4600)$ & $\rightarrow D(4)$ or $D(5)$ & $0.83,0.17$ \\
\hline 2 & $J(\mathrm{LL})$ and $R(0)$ and $r(0.34)$ and $W(\mathrm{M} \mathrm{Tig})$ and $M(\mathrm{JIS} \mathrm{H4600)}$ & $\rightarrow D(4)$ or $D(3)$ & $0.86,0.14$ \\
\hline 3 & $J(\mathrm{LT})$ and $R(0)$ and $r(0.27)$ and $W(\mathrm{M} \mathrm{Tig})$ and $M(\mathrm{JIS} \mathrm{H} 4600)$ & $\rightarrow D(3)$ or $D(4)$ & $0.83,0.17$ \\
\hline 4 & $J(\mathrm{LT})$ and $R(0)$ and $t(2)$ and $W(\mathrm{M} \mathrm{Tig})$ and $M(\mathrm{JIS} \mathrm{H} 4600)$ & $\rightarrow D(4)$ or $D(5)$ & $0.83,0.17$ \\
\hline 5 & $J(\mathrm{LL})$ and $R(0)$ and $t(2)$ and $W(\mathrm{MI}$ Tig) and $M(\mathrm{JIS} \mathrm{H} 4600)$ & $\rightarrow D(4)$ or $D(3)$ & $0.86,0.14$ \\
\hline 6 & $J(\mathrm{LT})$ and $R(0)$ and $t(10)$ and $W(\mathrm{M} \mathrm{Tig})$ and $M(\mathrm{JIS}$ H4600) & $\rightarrow D(3)$ or $D(4)$ & $0.83,0.17$ \\
\hline
\end{tabular}

Table 4. Reduction rules of S-N distribution based on NS

\begin{tabular}{|c|c|c|c|}
\hline No. & Rules & & Supports \\
\hline 1 & $R(0)$ and $r(0.29)$ and $W(\mathrm{M}$ Tig) and $M(\mathrm{JIS} \mathrm{H} 4600)$ & $\rightarrow D(3)$ or $D(4)$ or $D(5)$ & $0.54,0.31,0.15$ \\
\hline 2 & $R(0)$ and $r(0.34)$ and $W(\mathrm{M} \mathrm{Tig})$ and $M(\mathrm{JIS} \mathrm{H} 4600)$ & $\rightarrow D(4)$ or $D(3)$ & $0.43,0.57$ \\
\hline 3 & $R(0)$ and $r(0.27)$ and $W(\mathrm{M} \mathrm{Tig})$ and $M(\mathrm{JIS} \mathrm{H} 4600)$ & $\rightarrow D(3)$ or $D(4)$ & $0.5,0.5$ \\
\hline 4 & $J(\mathrm{LT})$ and $R(0)$ and $t(2)$ and $W(\mathrm{M} \mathrm{Tig})$ and $M(\mathrm{JIS} \mathrm{H} 4600)$ & $\rightarrow D(3)$ or $D(4)$ or $D(5)$ & $0.33,0.5,0.17$ \\
\hline 5 & $J(\mathrm{LL})$ and $R(0)$ and $t(2)$ and $W(\mathrm{Ml} \mathrm{Tig})$ and $M(\mathrm{JIS} \mathrm{H} 4600)$ & $\rightarrow D(4)$ or $D(3)$ & $0.43,0.57$ \\
\hline 6 & $J(\mathrm{LL})$ and $R(0)$ and $t(10)$ and $W(\mathrm{M} \mathrm{Tig})$ and $M(\mathrm{JIS}$ H4600) & $\rightarrow D(3)$ or $D(4)$ & $0.5,0.5$ \\
\hline
\end{tabular}


from $D(1)$ to $D(6)$. There are also large deviations in the $S-N$ data of $D(1)$ and $D(6)$ and small ones in $D(3)$ and $D(4)$. Parts of the $S-N$ data attributes' values in the rough set model based on NS are shown as an example in Table 1.

\section{RESULTS AND DISCUSSIONS}

With three welding $S-N$ data distribution rough set models based on $N S, S S$ and $E S$ established in the above procedure, reductions in the genetic algorithm were carried out for the $S-N$ data distribution models including all the factors. To obtain universal laws the reduction results were filtered with the support range removed from 0 to 0.75 and coverage from 0 to 0.05 .

Two reduction results from the rough set model based on $N S$ and $S S$ were obtained as $\{J, R, r, W$, $M\},\{J, R, t, W, M\}$ including 36 rules. After filtering 4 rules based on NS and 6 based on SS remained as indicated in Tables 2 and 3. Comparing the two tables, rule 1 and 3 in Table 1 are the same as rule 1 and 4 in Table 2. The decision attributes in rules 2 and 4 are changed from $D(3)$ or $D(2)$ to $D(3)$ or $D(4)$. As indicated in Fig. 2 the decision domains 3 and 4 are next to the mean $S$ - $N$ curve. In addition, two new rules exist in Table 3 whose decision attributes are both $D(3)$ or $D(4)$. So, this shows that the $S-N$ distribution based on $S S$ is more concentrated near the mean $S-N$ curve.

Two reduction results from $E S$ as $\{R, r, W, M\},\{J$, $R, t, W, M\}$ including 34 rules were also investigated. But no rules remained after filtering within the same parameters. To analyze the correction of the $S-N$ distribution from ES, similar rules to the ones in Table 3 were extracted as shown in Table 4. Comparing the rules from $E S$ and $S S$ it was found that the condition attribute of $J$ is eliminated in rules 1 to 3 . This means that the decision-making degree of factor $J$ is weakened to some extent. Besides decision attributes $D(3)$ with supports of 0.51 and 0.33 are added in rules 1 and 4 . The supports also change from $0.86,0.14$ to $0.43,0.57$ in rules 2 and 5 , from $0.83,0.17$ to 0.5 , 0.5 in rules 3 and 6 . Thus the supports of $D(3)$ and $D(4)$ become closer to each other, which implies that the $S-N$ data in decision domains 3 and 4 distribute more uniformly on both sides of the mean $S-N$ curve based on ES. So this makes the STDEV of the $S-N$ distribution smaller for $E S$ than for $N S$ and $S S$.

As discussed above, the structural stress rearranges the $S-N$ point so that it is much more concentrated in the region near the mean $S-N$ curve and the equivalent structural stress places it more uniformly on both sides of the curve. According to the stress calculation in the Master $S$ - $N$ curve method in Section 1, correction by $t$ and the welded structure in the structural stress calculation in Eq. 4 is first carried out. Then the structural stress correction using both $t$ and $r$ in the equivalent structural stress in Eq. 7 is carried out. With the two steps of correction the concentration and uniformity degrees of $S-N$ distribution are enhanced, which creates a Master $S-N$ curve with a smaller deviation.

From the results it was also found that the decision-making degrees of welding factors were weakened in the Master $S$ - $N$ curve method. To study this effect welding factors $t$ and $r$ were chosen to be analyzed because the stress was corrected mainly by the two parameters in the Master $S-N$ curve method. Therefore, the fatigue data based on NS, SS and ES in single series fatigue data with same $t$ and $r$ were analyzed. First, the STDEV of single series in $t$ and $r$ were calculated as shown in Tables 5 and 6. From Table 5 it can be seen that the STDEV decreases when the stress type changed from $N S$ to $S S$ in every $t$ and that this happens again from $S S$ to $E S$. According to Eq. 4 in the transformation process of $N S$ to $S S$ for a $3 \mathrm{D}$ welded structure, the stress is corrected by $t$ and the welded structure. Comparing the STDEV in stress type of $N S$ and $S S$ with the same $t$ it was concluded that the influence of the welded structure could also be corrected in the $S S$ transformation. From Table 6 it can be seen that the STDEV clearly decreases from NS to $S S$ in every $r$ but that no significant change appears from $S S$ to $E S$.

Table 5. STDEV of fatigue data in $t$ series

\begin{tabular}{cccccc}
\hline Stress Type & $t=1.6$ & $t=2$ & $t=2.5$ & $t=5$ & $t=10$ \\
\hline$N S$ & 0.93 & 0.41 & 0.41 & 0.59 & 0.53 \\
\hline$S S$ & 0.85 & 0.29 & 0.40 & 0.51 & 0.44 \\
\hline$E S$ & 0.73 & 0.19 & 0.40 & 0.43 & 0.34 \\
\hline Points & 19 & 20 & 35 & 12 & 20 \\
\hline
\end{tabular}

Table 6. STDEV of fatigue data in $r$ series

\begin{tabular}{cccccc}
\hline Stress Type & $r=0$ & $r=0.27$ & $r=0.29$ & $r=0.32$ & $r=0.34$ \\
\hline NS & 0.74 & 0.21 & 0.22 & 0.74 & 0.19 \\
\hline SS & 0.67 & 0.20 & 0.20 & 0.66 & 0.18 \\
\hline ES & 0.66 & 0.20 & 0.20 & 0.64 & 0.18 \\
\hline Points & 52 & 16 & 13 & 18 & 7 \\
\hline
\end{tabular}

To study the correction mechanism in more detail, two rough set models were rebuilt with only one condition attribute of $t$ or $r$ being kept and others being removed. Reductions were carried out within all the rules without filtering. The maximum values of supports that characterized the decision-making 
degrees were recorded at each condition attribute value of $t$ and $r$.

Figs. 5 and 6 indicate the maximum supports for $t$ and $r$ based on NS, SS and ES. From the results the decision-making degrees of $t$ and $r$ are somewhat larger based on NS than the other ones.

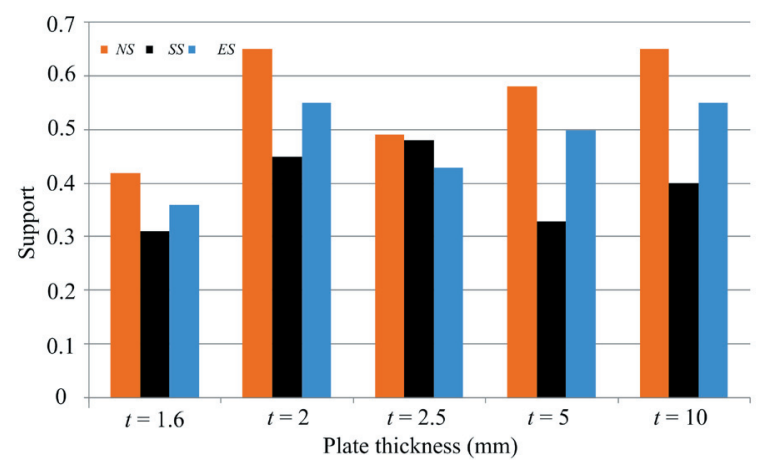

Fig. 5. Maximum support of condition attribute of $t$ based on NS, SS and ES

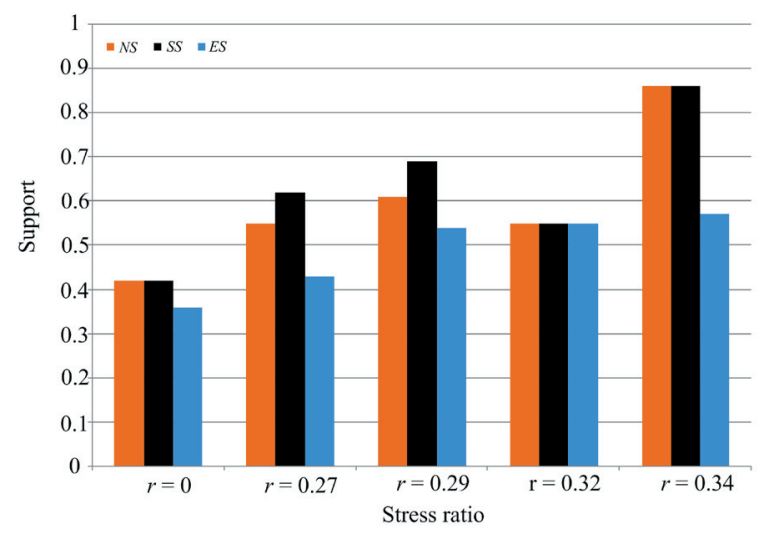

Fig. 6. Maximum support of condition attribute of $r$ based on NS, SS and ES

This demonstrates that the $S-N$ distribution based on nominal stress is easily affected by welding factors. From Fig. 5 where $t$ equals 1.4, 2, 5 and 10, the maximum supports based on $S S$ were greatly reduced compared to NS. However, Fig 6 shows that the maximum supports based on $S S$ are a little higher than the one based on NS. According to Eq. 4 and Table 5 , the stress is corrected mainly by $t$ without $r$ in structural stress. This is the reason that the supports based on $S S$ weaken greatly for $t$ but are enhanced for $r$. The results from $E S$ in Fig. 5 showed that, compared to $S S$, the maximum support of $t$ increases to a certain degree, but is still less than the that for NS. Fig. 6 shows that the obvious reduction in the maximum supports of $r$ based on ES can be observed compared to the other two especially in the case of $r$ equal to
0.34. The two histograms show that the differences between the maximum supports for all cases are narrowed. Combined with Table 6, this proves that the decision-making degrees of welding factors are weakened and harmonized in the Battelle equivalent structural stress. The deviations of $S-N$ data from the mean $S-N$ curve are compensated for by the two steps of correction using structural stress and equivalent structural stress, which makes the $S-N$ distribution much more concentrated and uniform. This allows for a more accurate welding fatigue prediction using just one $S-N$ curve to represent the complete $S-N$ data set.

\section{CONCLUSIONS}

Rough set theory was employed to study the $S-N$ distribution based on the Battelle equivalent structural stress in the Master $S-N$ curve method. Rough set models of Titanium alloy welding joints' $S-N$ distribution were established based on nominal stress, the structural stress and Battelle equivalent structural stress. Rough set analyses based on the three kinds of stresses were then carried out using the welding $S-N$ distributions studied. The results indicated that the $S T D E V$ decreased gradually in the transformation of stress type from NS to ES. The structural stress rearranges the $S-N$ point so that it is much more concentrated in the region near the mean $S-N$ curve and the equivalent structural stress places it more uniformly on both sides of the curve. Subsequently the correction of the $t$ and $r$ factors in the Master $S-N$ curve method were studied. From the results it was concluded that with these two steps of correction, the decision-making degrees of welding factors are weakened and harmonized in the Battelle equivalent structural stress. This makes the $S-N$ distribution much more concentrated and uniform, which allows for a more accurate welding fatigue prediction by the Master $S-N$ curve.

\section{ACKNOWLEDGEMENT}

The work reported on in this paper was supported by the National Natural Science Foundation of China (Grant No. 51175054) and the Science and Technology Plan project of Liaoning (Grant No. 2011220039).

\section{REFERENCES}

[1] Fricke, W. (2003). Fatigue analysis of welded joints state of development. Marine Structures, vol. 16, no. 3, p. 185-200, DOI:10.1016/S0951-8339(02)00075-8. 
[2] BS7608-1993. (1999). Fatigue Design and Assessment of Steel Structures. The British Standards Institution, London.

[3] IIS/IIW-1221-93 (1995). Stress Determination for Fatigue Analysis Welded Components. The International Institute of Welding, Abington Publication, Cambridge.

[4] Niemi, E. (1994). On the Determination of Hot Spot Stresses in the Vicinity of Edge Gussets. IIW Document XIII-1555-94. Lappeenranta University of Technology, Department of Mechanical Engineering. Lappeenranta, p. 18.

[5] Niemi, E., Tanskanen, P. (2000). Hot spot stress determination for welded edge gussets. Welding in the World, vol. 44, no. 5, p. 31-37.

[6] Lotsberg, I., Sigurdsson, G. (2006). Hot spot stress S-N curve for fatigue analysis of plated structures. Journal of Offshore Mechanics and Arctic Engineering, vol. 128 , no. 4, p. 330-336, DOI:10.1115/1.2355512.

[7] Dong, P. (2001). A structural stress definition and numerical implementation for fatigue analysis of welded joints. International Journal of Fatigue, vol. 23, no. 10, p. 865-876, DOI:10.1016/S01421123(01)00055-X.

[8] Dong, P., Hong, J.K. (2004). The Master S-N curve approach to fatigue of piping and vessel welds. Welding in the World, vol. 48,no. 1-2, p. 28-36, DOI:10.1007/ BF03266411.

[9] ASME (2007). 2007 ASME Boiler and Pressure Vessel Code Section VIII Division 2 Part 5: Design by Analysis Requirement. The American Society of Mechanical Engineers, New York.
[10] API (2007). API 579-1/ASME FFS-1 2007 Fitnessfor-Service. The American Petroleum Institute, Washington.

[11] Paris, P.C. (1998). Fracture mechanics and fatigue: a history perspective. Fatigue \& Fracture of Engineering Materials \& Structures, vol. 21, no. 5, p. 535-540, DOI:10.1046/j.1460-2695.1998.00054.x.

[12] Pawlak, Z. (1982). Rough set. International Journal of Computer and Information Science, vol. 11, p. 341356, DOI:10.1007/BF01001956.

[13] Qi, Y., Deng, J., Hong, Q., Zeng, L. (2000). Electron beam welding, laser beam welding and gas tungsten arc welding of titanium sheet. Material Science and Engineering A, vol. 280, no. 1, p. 171-181.

[14] Oh, J., Kim, N., Lee, S., et al. (2003). Correlation of fatigue properties and microstructure in investment cast Ti-6Al-4V welds. Material Science and Engineering, A, vol. 340, no. 1-2, p. 232-242, DOI:10.1016/S09215093(02)00176-4.

[15] Casavola, C., Pappalettere, C., Tattoli, F. (2009). Experimental and numerical study of static and fatigue properties of titanium alloy welded joints. Mechanics of Materials, vol. 41, no. 3, p. 231-243, DOI:10.1016/j. mechmat.2008.10.015.

[16] Iwata, T., Matsuoka, K. (2004). Fatigue strength of CP Grade 2 Titanium fillet welded joint for ship structure. Welding in the World, vol. 48, no. 7-8, p. 40-47, DOI:10.1007/BF03266442.

[17] Mohr, W., Lawmon, J. (2006). Fatigue testing of structural welds for Titanium alloy structures. Proceedings of the $16^{\text {th }}$ International Offshore and Polar Engineering Conference, San Francisco, p. 137144. 